Trans

continentales
Transcontinentales

Sociétés, idéologies, système mondial

$10 / 11 \mid 2011$

La ruée vers la terre

\title{
Délocalisation et dys-localisation dans le foncier contemporain
}

Delocalization and dyslocalization in land today

\section{Gérard Chouquer}

\section{OpenEdition}

\section{Journals}

Édition électronique

URL : http://journals.openedition.org/transcontinentales/1071

DOI : 10.4000/transcontinentales.1071

ISBN : 978-2-7351-1572-3

ISSN : $1775-397 X$

\section{Éditeur}

Editions de la maison des sciences de l'homme

Référence électronique

Gérard Chouquer, "Délocalisation et dys-localisation dans le foncier contemporain »,

Transcontinentales [En ligne], 10/11 | 2011, document 2, mis en ligne le 19 octobre 2011, consulté le 08 septembre 2020. URL : http://journals.openedition.org/transcontinentales/1071 ; DOI : https://doi.org/ 10.4000/transcontinentales.1071

Ce document a été généré automatiquement le 8 septembre 2020

Tous droits réservés 


\title{
Délocalisation et dys-localisation dans le foncier contemporain
}

\author{
Delocalization and dyslocalization in land today
}

\author{
Gérard Chouquer
}

1 L'avenir du foncier dans le monde se joue en ce moment. Non pas que la pression commerciale sur les terres soit neuve : elle existait déjà avec les concessions coloniales de l'époque moderne. L'originalité de la situation actuelle est matérialisée par des formes nouvelles d'appropriation qui pourraient être amenées à se généraliser. Leurs fondements juridiques, économiques et sociaux sont basés sur le principe d'une délocalisation systématique, c'est-à-dire d'une dissociation de tout ce que les lieux, jusqu'ici, réunissaient : la terre, les hommes, les productions, les moyens. J'illustrerai mon propos à travers l'analyse de deux exemples très différents. Je m'appuierai sur les cas des projets Varun à Madagascar, et Yasuni en Équateur. Le premier a largement été déprécié dans la presse et correspondrait donc à une version contestée tandis que le second a bénéficié d'une couverture médiatique relativement favorable illustrant une vision positive de ce type de processus.

2 Je souhaite montrer dans cet article que, malgré leur différence, la nature agéographique $\mathrm{du}$ dispositif est identique et repose sur un principe identique, la délocalisation.

\section{Le projet Varun à Madagascar}

3 Succédant à la révélation du projet de Daewoo, un autre vaste projet d'investissement dans les terres agricoles par la firme indienne Varun International à Madagascar a suscité de nombreux débats aussi bien à l'échelon malgache que dans le monde entier. Le gouvernement malgache devait céder des surfaces considérables de terres arables à ces entreprises étrangères, lesquelles cherchaient des réponses aux besoins commerciaux et alimentaires de leurs pays surpeuplés (Corée du Sud et Inde). Dans le 
cas du projet Varun, il s'agissait égalementde s'inspirer explicitement des théories néolibérales.

Le contrat envisagé entre Varun International et le gouvernement malgache était un projet d'agriculture contractuelle (contract farming) ${ }^{1}$, qui concernait 465000 hectares et la création de treize périmètres irrigués permettant de produire 2,8 millions de tonnes de riz paddy et 400000 tonnes de maïs dès la quatrième année.

5 Le protocole signé, en octobre 2008, entre la société Varun et la région de Sofia comportait des indications précises concernant les relations entre les différents acteurs de ce nouveau processus.

6 Tout d'abord, Varun négociait avec deux sortes d'intermédiaires chargés d'établir et de régler les relations avec la population locale. Les premiers étaient treize collectivités ou instances gérant chacune un périmètre irrigué et fournissant à Varun l'assurance de la disponibilité des terrains comme de la faisabilité des projets. Le second, un bureau de conseil malgache (SODHAI) ${ }^{2}$, devait réaliser les négociations avec les " plaines » et les paysans pour la mise en œuvre des contrats. SODHAI est encore de nos jours le partenaire local qui réalise divers projets de développement agricole avec des sociétés indiennes.

7 Pour éviter des conflits potentiels générés par la présence de population sur les terres qui avaient pourtant été déclarées vacantes par l'administration, Varun souhaitait généraliser une procédure de contractualisation que SODHAI et les treize "plaines " formalisaient avec les paysans habitant les futurs périmètres des contrats de production agricole.

8 Le caractère inique du projet Varun apparaît lorsqu'on observe certaines clauses que les paysans devaient accepter. Le contrat prévoyait notamment que les paysans n'engagent pas de revendications contre la société indienne. SODHAI apparaissait comme l'unique interlocuteur pour pacifier les relations avec les paysans afin de garantir à Varun une exploitation directe et sans entrave. Ensuite, Varun prévoyait de commercialiser les produits agricoles en fonction d'un contrat très clair et correspondant à ses intérêts : 70 \% de la production aurait dû revenir à Varun, et sur les $30 \%$ restant au détenteur des terrains (par grant, c'est-à-dire par libéralité gracieuse de Varun), $70 \%$ devait obligatoirement être vendu à Varun, au cours fixé par l'entreprise. Autrement dit, sur une tonne produite par le paysan ayant un contrat avec la firme, l'entreprise indienne se réservait d'abord 700 kilos puis, sur les 300 autres kilos, le producteur devait en vendre 210 à Varun au prix fixé par celle-ci. Varun contrôlait ainsi 910 kilos, soit $91 \%$ de la production. Certains spécialistes ont calculé que de telles clauses auraient obligé les modestes producteurs malgaches à acheter du riz pour se nourrir...

9 Un tel type de contrat entraîne toute une série d'interrogations concernant l'évolution de l'agriculture et de l'alimentation à l'échelle planétaire.

10 Varun International est un acteur de l'industrie agroalimentaire indienne implanté à Bombay. Sa spécialité n'est pas l'agriculture, mais le grand commerce maritime. Ses activités couvrent différents secteurs comme le commerce des marchandises, la fabrication de produits manufacturés métallurgiques mais aussi l'exploitation minière (or, platine, uranium). Varun cherche également à développer de nouveaux secteurs, comme l'exploitation du gaz, du pétrole et de l'énergie éolienne. Sa filiale malgache, Varun International Madagascar SARL, assure l'import-export de marchandises 
générales et de produits miniers. Cette implantation antérieure sur le sol malgache peut expliquer en partie le choix d'une diversification d'activités.

11 Les termes du contrat évoquent notamment le développement de nouveaux secteurs d'activité tel l'aménagement rural de périmètres irrigués, mais aussi le lotissement urbain amenant à la création de villes nouvelles.

12 Ces entreprises aux activités économiques diversifiées saisissent, en choisissant d'investir dans l'agrobusiness, des opportunités commerciales et financières générées par le marché de leurs pays d'origine. Daewoo et Varun importent, dans leur propre pays, des produits alimentaires pour satisfaire les besoins de leur population mais aussi des produits agricoles à destination énergétique comme les agrocarburants, pour pallier leur manque de pétrole.

13 On peut se demander, quand bien même la légalité du projet aurait été assurée, s'il est légitime d'exploiter des terres dans un pays qui n'est pas autosuffisant en matière d'alimentation afin d'exporter les produits agricoles dans un autre pays? En effet, à Madagascar, le Programme alimentaire mondial de l'ONU aide environ 500000 paysans chaque année et $70 \%$ de la population vit en dessous du seuil de pauvreté. Il existe donc une disproportion considérable de nature, de niveau et d'objectifs entre la réalité économique et sociale des campagnes malgaches et les projets envisagés.

14 Ce problème apparaît avec une acuité particulière lorsque l'investissement concerne un pays très pauvre, dépendant du Programme alimentaire mondial. S'il s'agit d'une conséquence économique de l'évolution du commerce mondial, ce processus apparait comme un non-sens philosophique et politique : des pays pauvres devant faire appel à l'aide alimentaire internationale exportent des matières premières agricoles dont la population aurait besoin.

Dans une excellente publication récente, Lorenzo Cotula $^{3}$ a proposé un schéma pour caractériser la nature de ce type de contrats d'acquisitions massives de terres qui tend à se généraliser à travers le monde. J'en donne ci-dessous une traduction (Fig. 1). 
Fig. 1

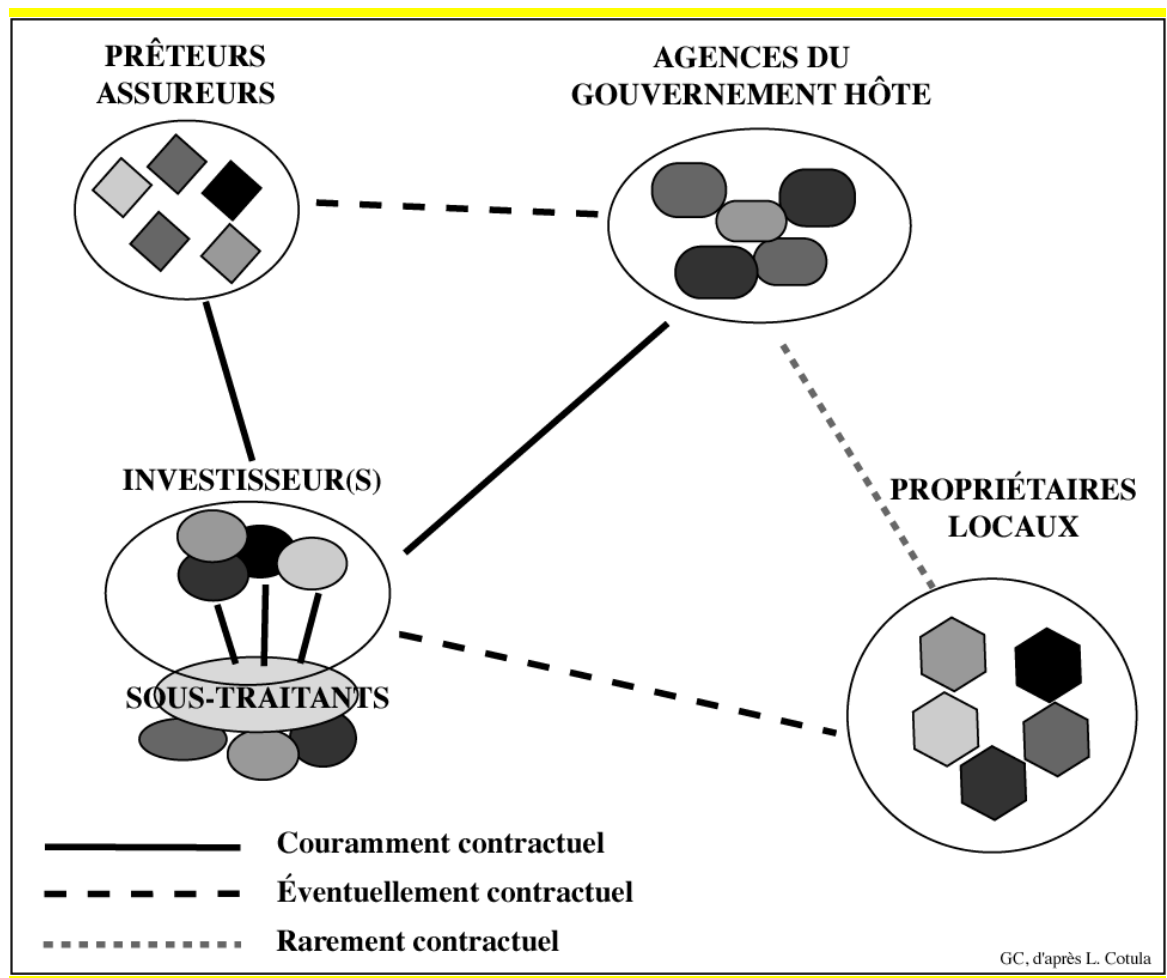

La structure des contrats d'après Lorenzo Cotula

Mais, dans la figure qui suit (Fig. 2), j'interprète le schéma du double triangle de relations de Lorenzo Cotula au moyen d'analogies avec des structures sociales et juridiques du droit foncier et commercial.

Fig. 2

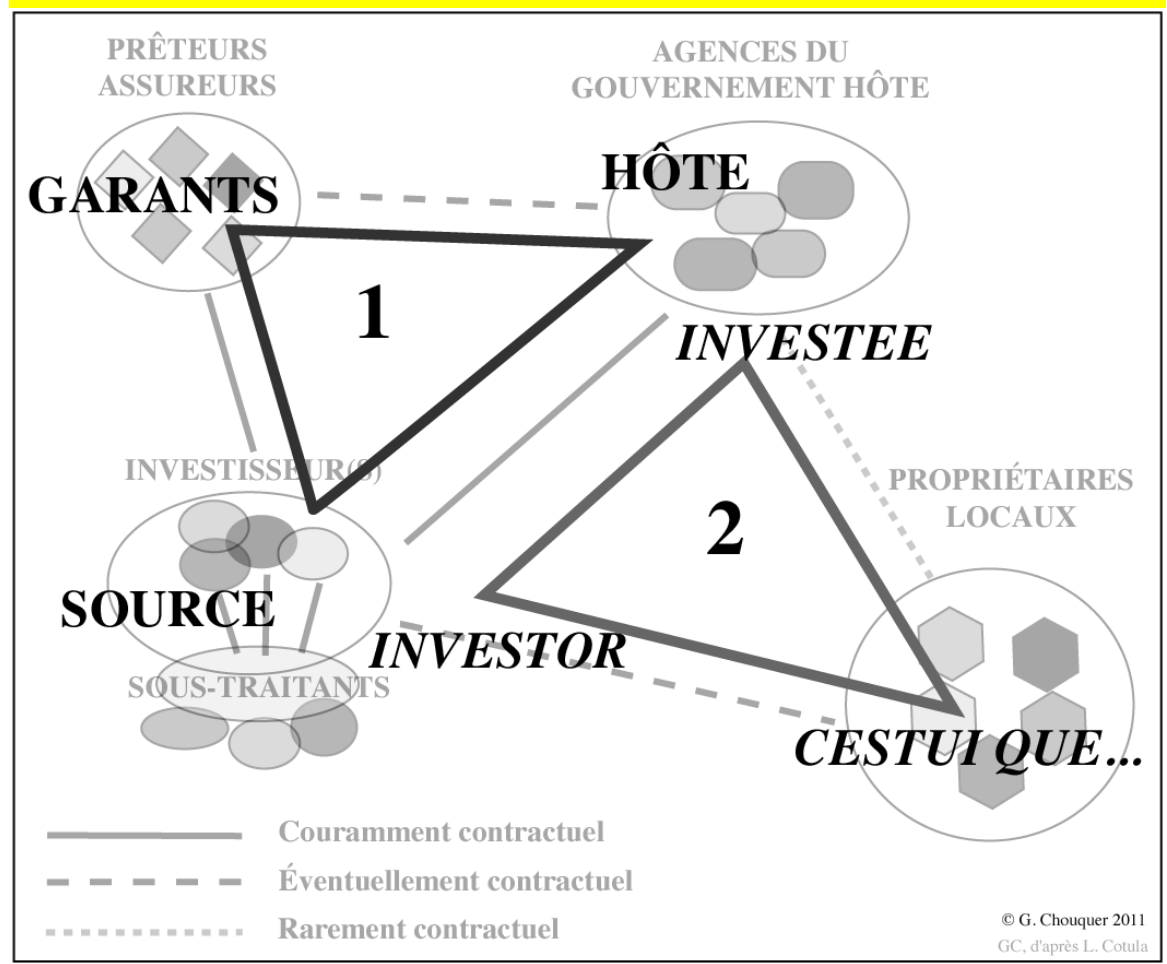

Interprétation du schéma de Lorenzo Cotula en termes de sociologie et de droit. 
Dans le premier triangle (noté 1 sur la figure 2), il est possible de reconnaître trois fonctions théorisées par les néolibéraux dans la théorie dite des Charter cities et que j'applique ici aux contrats sur les terres. Partant d'un constat de besoins en espace, en nourriture, ou en ressources dans certaines parties du monde (fonction "source»), et de disponibilités en terres dans d'autres (fonction "hôte»), cette école propose une contractualisation à l'échelle mondiale, sous la garantie d'institutions ou de pays tiers (fonction de "garant»). Cette contractualisation volontaire passerait par la création de villes nouvelles servant de portes d'entrée dans les pays hôtes.

17 En adaptant ce schéma aux acquisitions massives de terres, je propose de parler de nouveau commerce triangulaire. La source désigne les pays et les investisseurs qui prennent l'initiative de nourrir leur propre population, de produire pour leur propre usage des agrocarburants ou, tout simplement, spéculent. L'hôte désigne les lieux, les États, les collectivités ou les organismes destinataires de la proposition d'acquisition, le plus souvent, par le biais des agences gouvernementales locales ou par celui des intermédiaires chargés de réaliser le projet. Enfin, la fonction de garant est assurée par les bailleurs de fonds (fonds d'investissement) ou les assureurs des projets, voire ceux qui apportent une garantie juridique : il peut s'agir de fonds des Nations unies (ex. : le PNUD), de gouvernements, de trusts, de fonds d'investissement souverains, etc.

Dans le second triangle (noté 2 sur la figure 2), je propose une autre analogie en identifiant une structure du type trust pour interpréter les relations qui se nouent entre investisseurs, pays hôte et population locale. Un trust est un contrat légal du droit anglais permettant de tourner des règles juridiques trop strictes (par exemple, celles du common law sur les droits de succession) en créant un intermédiaire pour gérer un bien pour le compte d'un ou plusieurs bénéficiaires désignés. Ainsi, selon le droit anglais, un propriétaire peut mandater un intermédiaire de confiance (d'où la notion de fiduciarité) pour administrer un bien et les intérêts de ses divers ayants droit plutôt que de le leur transmettre directement. La personne qui initie ou constitue le trust est appelée le trustor ou settlor; celle à qui il remet le bien est le trustee ou settlee; enfin, le bénéficiaire est dit cestui que trust, celui qui est l'objet du trust. Au-delà des avantages fiscaux recherchés, l'originalité du montage vise à rendre le lien au patrimoine un peu plus virtuel, puisque le même bien est chargé de plusieurs droits de propriété. En effet, le trustee se voit réellement reconnu comme propriétaire et non juste comme gestionnaire du bien, et le futur bénéficiaire (par exemple l'héritier) voit son (futur) droit de propriété redéfini : on dit alors qu'il a un «intérêt » (estate en droit anglais), qui est en outre différé. Le même bien relève donc de plusieurs "propriétaires » ou formes de propriété: le constituant qui garde un droit dessus; l'intermédiaire fiduciaire qui en est investi ; le bénéficiaire à qui est reconnu un droit ou intérêt différé.

On comprend que la forme du trust ait, depuis le XIX ${ }^{e}$ siècle, séduit les investisseurs par sa souplesse. Le mot trust a d'ailleurs évolué dans sa définition et il est aujourd'hui surtout connu dans son sens économique : le trust est une entreprise qui a obtenu une situation de monopole et qui, pour réaliser la concentration à son profit et pour dicter sa loi aux entreprises du même secteur, a utilisé la forme juridique du trust. Sans absorber purement et simplement les entreprises concurrentes qu'elle a fragilisées, elle organise un montage qui permet de les gérer dans une forme commune fiduciaire.

20 C'est ce qu'a fait Varun International à Madagascar. Plutôt que de négocier avec les occupants locaux, cette entreprise traite avec treize "plaines ", collectivités locales retenues dans ce but, et avec un bureau spécialisé, SODHAI. Le schéma, bien que le 
propos soit différent, reproduit une relation fiduciaire du type trust : l'intermédiaire (investee) se substitue complètement aux populations locales (cestui que invest - ceux qui sont l'objet de l'investissement) en prenant en charge, au moins en théorie, leur «intérêt " lointain en tant que bénéficiaires du projet. En effet, le projet doit, en principe, leur donner du travail, leur apporter des équipements, favoriser le commerce local, etc. Quant à Varun, elle remplit bien le rôle de source, d'initiateur ou investor du projet.

\section{Le projet Yasuni en Équateur}

Le second exemple est le projet Yasuni-ITT ${ }^{4}$ dans le parc amazonien de Yasuni en Équateur. Il est question de geler l'exploitation du pétrole dans cet espace à la biodiversité unique au monde, à condition que l'État équatorien soit dédommagé des pertes substantielles que le renoncement à l'exploitation pétrolière lui ferait subir. Au terme d'un long débat public, les autorités politiques ont en effet indiqué que le pays accepterait d'y renoncer, et de contribuer à la coresponsabilité environnementale globale si la communauté internationale offrait une compensation financière ${ }^{5}$. Des pays ou des institutions internationales ont été invités à assurer ce dédommagement.

La proposition de mise en réserve du secteur ITT a été rendue publique, en juin 2007, par le président Rafael Correa. Le mécanisme proposé consistait à encourager la communauté internationale à contribuer à ce projet pour que le gouvernement équatorien limite ses émissions de gaz à effet de serre en n'exploitant pas les réserves du secteur ITT. L'État équatorien proposa de vendre des bons Yasuni-ITT, en garantissant un remboursement dans le cas où un gouvernement ultérieur renoncerait à cet engagement écologique.

23 La fin de l'année 2011 a été fixée comme date butoir par les autorités équatoriennes pour rassembler les diverses contributions, faute de quoi il procédera à la mise en exploitation des gisements pétrolifères. Jusqu'à présent, le fonds international de garantie a enregistré la participation financière du Chili, de l'Espagne, de la Région de Wallonie (Belgique) et de la fondation latino-américaine Avina. D'autres pays ont manifesté leur intérêt comme le Japon, la Norvège, le Portugal, le Canada, l'Allemagne (une promesse de 50 millions d'euros), l'Italie, le Pérou, la Turquie, les Émirats arabes unis, sans avoir cependant signé d'engagement ferme. Le Programme des Nations unies pour le développement (PNUD) a offert sa caution.

24 L'accord prévoit que le capital du Fonds Yasuni-ITT serve au développement de sources renouvelables. Le fonds devrait financer des projets contre la déforestation et pour la conservation de 44 aires protégées (sur 4,8 millions d'hectares soit $20 \%$ du territoire équatorien); pour reforester et régénérer un million d'hectares de forêt dégradée; pour soutenir le développement social (éducation, santé, habitat, création d'emplois soutenables comme ceux de l'écotourisme); et investir dans la recherche et le développement en sciences et technologies de la bioconnaissance.

La fiduciarisation dont ce projet est porteur se traduit par une double triangularisation des relations (Fig. 2).

26 Le premier triangle reproduit un schéma du type source-hôte-garant. La source est ici la pression multiforme (internationale et nationale) pour faire respecter, à la fois, les droits des populations autochtones, la protection de la biodiversité et la contribution à 
la lutte contre le réchauffement climatique. L'hôte est évidemment l'espace du parc Yasuni, objet de la pression internationale et que le gouvernement équatorien exploite avec intelligence en faisant valoir le caractère alternatif de la solution qu'il propose. Enfin, les garants sont les États et les ONG qui s'engagent financièrement afin de constituer le fonds dont l'Équateur a fixé le montant.

Mais un second triangle se constitue en parallèle, comme garantie de la garantie. Dans le cas du projet Yasuni, il met en jeu les bailleurs de fonds (la source), le fonds pour l'utilisation de cette somme en Équateur (l'hôte) et la sécurité qu'offre le Programme des Nations unies pour le développement (le garant), à la suite de l'accord entre le gouvernement équatorien et le PNUD.

Il apparaît clairement que le montage d'un tel projet porte atteinte à la souveraineté équatorienne. Cette question a été en grande partie l'objet d'un conflit entre le président Correa et certains de ses ministres et conseillers, au début de 2010. L'autre élément du conflit résidait dans le désir des écologistes radicaux de l'entourage du président Correa de «mettre fin à l'exploitation d'hydrocarbures et de ressources minières dans la moitié du pays ${ }^{6} "$, soit sur une surface bien plus importante que dans le seul bloc ITT. On note également que le sort d'un espace sanctuarisé pourrait être remis à un consortium extérieur. Que se passerait-il si les bailleurs de fond internationaux se retiraient?

Avant de conclure sur les déviances de la délocalisation et de la fiduciarisation des relations, il faut relever les aspects positifs, voire novateurs, que le projet Yasuni-ITT a mis en avant. Malgré les hésitations sur le montage du projet, pour la première fois, une réflexion a été menée pour imaginer des mécanismes financiers et économiques alternatifs à ceux fondés sur les valeurs marchandes dominantes. Ensuite, on a vu se développer en Équateur un processus de délibération sans précédent dans ce type de pays. Enfin, comme le relève Boaventura de Sousa Santos, «la proposition combine les préoccupations environnementales occidentalo-centristes et le respect pour les conceptions indigènes de la Pachamama (la Terre Mère). Elle justifie le droit de la nature à être protégée en tant qu'entité vivante chaque fois que la stabilité et la régénération de ses cycles vitaux sont menacées. Elle proclame l'idée de Sumak Kawsay, le «bienvivre ", comme une alternative aux conceptions occidentales du développement (toutes considérées comme non-durables) et à la croissance infinie. Elle doit donc être estimée comme une contribution indigène au monde entier ${ }^{7}$ ».

\section{Un dysfonctionnement : le lieu n'est plus le lien}

Ces deux exemples sont en quelque sorte des situations virtuelles car le projet Varun semble avoir été abandonné tandis que le projet Yasuni semble de moins en moins certain. Ils sont révélateurs de nouvelles dynamiques qui risquent de se généraliser dans un avenir proche. Dans ces deux situations très différentes, on constate cependant un principe de délocalisation qui conduit à un dysfonctionnement de la localisation que je qualifie pour cette raison de "dys-localisation». Il est assez difficile de penser que les situations foncières de zones importantes du monde puissent se construire sur cette base, c'est-à-dire en disjoignant de façon aussi forte la réalité économique de la réalité ontologique et géographique, sans que cela entraîne des conséquences politiques et sociales graves. Que penser de la qualité de la relation avec le milieu lorsque des institutions commerciales et financières s'approprient des lieux où leurs actionnaires 
et clients n'habiteront jamais, tandis que des gens qui les habitent se voient dépossédés d'une grande part de ce qui fait leur relation au lieu, c'est-à-dire le pouvoir de décider de leur avenir, celui d'assurer la maitrise foncière et usufruitière de leurs terres, de choisir la forme de leur labeur, etc. ?

Le rapport qui unit la terre d'un lieu et les hommes qui l'habitent et l'exploitent évoque des attaches profondes. Le lieu donne une identité géographique, mais aussi une mémoire parce qu'il assure des formes de transmission du passé qui relient les hommes à travers les générations. Nous venons de temps anciens où la mobilité était plus réduite, en tout cas, très différente. L'attachement des hommes aux lieux était constitutif du mode d'existence. On " était », c'est-à-dire qu'on existait, parce qu'on vivait quelque part et pas n'importe où, on était de ce lieu. Aujourd'hui, parce que la mobilité s'est généralisée, on n'est guère plus qu'originaire d'un lieu, car il est de plus en plus rare d'habiter son lieu de naissance.

Comme l'a observé le géographe Augustin Berque, cette force de la localisation géographique, cet apport que le lieu donne à la construction de l'identité individuelle et collective, peut se dire, dans le langage courant, par un mot simple, le plus réduit qui soit quand il s'agit du "y». J'y suis, j'y habite, j'y vais, dit-on pour exprimer le fait d'être en ce lieu qu'on désigne, qu'on habite, ou dans lequel on se rend. Ainsi, on " est » parce qu'on est là. On ne " serait » pas si on n'était pas quelque part, on n'existerait pas sans un lieu de référence. Augustin Berque l'exprime de façon abstraite en disant qu'on n'aurait pas d'ontologie si on n'avait pas une géographie. D'autres mots courts, encore, et presque passe-partout, si l'on peut dire, l'expriment : en (j'en viens), là, ici.

Les hommes ont donné des noms aux lieux, larges ou circonscrits, ambitieux ou anecdotiques, peu importe. La mémoire de ces façons de les dire - on pourrait inverser et parler de « dits de lieux » au lieu de « lieux-dits »- contribue à façonner l'identité.

Dans le rapport à la terre, les hommes du passé avaient découvert une façon de dire leur façon d'être. Ils jaugeaient l'espace à la mesure du corps, l'évaluant par des pas, des coudées, des brasses, des pieds; l'orientaient en se situant par rapport aux points remarquables qu'un lieu offrait, puisque d'un lieu à l'autre ces points changeaient. Innombrables sont les analogies que les anciennes communautés ont su développer pour dire la raison des lieux, ou mieux, les raisons de leurs lieux.

Or, même si cette raison diffère parfois de la nôtre, nous devinons combien cette somme d'associations entre dans la composition de la mémoire des lieux. Et nous aspirons tous, plus ou moins, à retrouver cette qualité de la relation au lieu de prédilection qui est le nôtre.

La manière dont l'homme agit aujourd'hui avec l'espace géographique constitue une rupture brutale de cette relation de l'« être au monde ", la plus radicale qui soit. Dans la logique triangulaire qui a été observée, on peut résumer en trois mots ce que deviennent les façons d'être au monde : délocalisation, mobilité, détachement.

Épargnants, spéculateurs, militants, producteurs, consommateurs, habitants, tous sont concernés par le nouveau mode de production agraire, et par la chaîne qu'il implique. Tous ont désormais un rapport différent aux lieux. Par exemple, depuis longtemps, le consommateur ne connaît plus d'où viennent les produits qu'il achète, si bien que l'affichage d'une origine locale est devenu un argument de qualité qui séduit. Depuis longtemps, les investisseurs pratiquent la mobilité et les producteurs délocalisent pour minimiser leurs coûts de production. Mais aujourd'hui la dissociation entre fonctionset 
lieux à l'échelle mondiale atteint un degré extrême que l'on retrouve dans la délocalisation de certains objets symboliques de certaines régions, ou dans des superficies et pour des besoins qui s'annoncent considérables. On peut ainsi dire : alors que le processus de détachement était déjà bien amorcé par les nouvelles formes de production, le phénomène des appropriations de terre à grande échelle achève d'installer le divorce entre les hommes et les milieux en ce qui concerne l'alimentation, l'énergie et le rapport à la terre.

La délocalisation et la mobilité impliquent un grand détachement. Pour que le système soit viable, certaines conditions sont déjà réunies: des capitaux mobiles, des entreprises internationales qui investissent en se redéployant sans cesse, des systèmes juridiques à deux ou même plusieurs vitesses. D'autres sont moins évidentes : des consommateurs lointains indifférents aux conditions de production de ce qu'ils consomment; des agriculteurs coupés du rapport à la terre et au lieu de production, devenus ouvriers d'un processus agricole ou d'élevage qu'ils ne contrôlent plus; des habitants eux-mêmes privés de la relation au milieu qui était, jusqu'ici, le leur.

Ce grand détachement prend les populations à revers. Il contredit également ceux des théoriciens qui, depuis quelque temps, élaborent des thèses attachantes pour expliquer ce que doit être la qualité de "l'être au monde» et la bonne façon de procéder. «Attachantes» est une allusion et un jeu de mot : chez les sociologues, les géographes et les anthropologues, ce terme désigne désormais un basculement épistémologique majeur, à la recherche de formes nouvelles de ré-attachement ou ré-enchantement du monde.

Nous vivons donc un changement. Alors que nous n'avons pas encore fait le décompte des méfaits des délocalisations, notamment industrielles, que le monde a connues, voilà qu'une variante nouvelle, la dys-localisation généralisée, se profile pour nous dire ce que sera le monde de demain. J'emploie le préfixe dys- pour signifier une anomalie de la localisation, un dysfonctionnement par rapport à ce que devrait être le bon rapport au lieu. La dys-localisation est une coupure de l'attache que nous avons aux lieux et à leurs qualités, doublée d'une série de défauts ou de faiblesses, quand il ne s'agit pas de crises. Dans dyslocalisation, je souhaite qu'on entende en écho délocalisation et dislocation. Une chose qui se disloque, perd la qualité de son lien au lieu; dis-locare en latin peut être traduit par désarticuler, déboîter, désunir, déranger, etc. Aujourd'hui, par les formes nouvelles de production, on atteint le degré de la plus grande dislocation. La géographie va-t-elle devoir devenir la médecine des lieux, diagnostiquant les pathologies et suggérant des remèdes qu'on ne suivra évidemment pas souvent?

41 La délocalisation ou dyslocalisation est la nouvelle mesure du monde. Jadis j'emprunte l'image à Bruno Latour (1997: 153) -, lors des épisodes coloniaux, pour prendre cette mesure, les Occidentaux ont opéré par le fer, le sang, la foi et le savoir, pour imposer leurs étalons, là où il en existait d'autres. Aujourd'hui, un des nouveaux critères de mesure est la virtualisation du rapport aux lieux pour permettre des formes souples de redéploiement. 


\section{BIBLIOGRAPHIE}

ANDRIANIRINA RATSIALONANA R. and A. Teyssier, « Large scale agricultural investments in Madagascar: lessons for more "inclusive" models ", in L. COTULA et R. LEONARD, Alternatives to Land Acquisition: agricultural land investments and collaborative business models, London/Rome/Berne/ Maputo, International Institute for Environment and Development(IIED)/International Fund for Agriculture Development (IFAD)/Swiss Agency for Development and Cooperation (SDC)/Centro Terra Viva (CTV), 2010 : 81-91.

ANDRIANIRINA RATSIALONANA R., L. RAMAROJOHN, P. BURNOD et A. TEYSSIER, « Après Daewoo ? État des lieux et perspectives des appropriations à grande échelle à Madagascar ", étude CIRAD/ Observatoire du foncier Madagascar, 2010 (version provisoire) [en ligne], consulté en octobre 2011.

URL : www.observatoire-foncier.mg/get-file.php?id=70

BERQUE A., Écoumène. Introduction à l'étude des milieux humains, coll. « Mappemonde », Paris, Belin, 2000.

Charter Cities : http://www.chartercities.org/home

CHOUQUER G., « Le nouveau commerce triangulaire ou les analogies du foncier contemporain », Études rurales, $\mathrm{n}^{\circ} 187,2011: 95-130$.

CотULA L., Land Deals in Africa: What is in the Contracts?, London, International Institute for Environment and Development (IIED), 2011.

COTULA L., S. VERMEULEN, R. LEONARD and J. KEELEY, Land Grab or Development Opportunity? Agricultural Investment and International Land Deals in Africa, London/Rome, IIED/FAO/IFAD, 2009.

DANIEL S. et A. MITTAL, (Mis)investment in Agriculture. The Role of the International Finance Corporation in Global Land Grab, préface de Howard G. Buffett, The Oakland Institute, 2010.

FONTAINE G., Gaz et pétrole en Amazonie. Conflits en territoires autochtones, L'Harmattan, Paris 2011.

LE QUANG M., « Une innovation dans la lutte contre le réchauffement climatique : l'initiative Yasuní-ITT en Équateur », Mouvements des idées et des luttes [en ligne], 19 septembre 2010, consulté en octobre 2011.

URL : http://www.mouvements.info/Une-innovation-dans-la-lutte.html\#nb23

MERLET M. et C. JAMART, avec la collaboration de M. PERDRIAULT et S. L'ORPHELIN, « Commercial Pressures on Land Worldwide. Issues and Conceptual Framework for International Land Coalition (ILC) study », ILC, mars 2009.

PERDRIAULT M. et M. MERLET, « Les appropriations de terres à grande échelle. Analyse du phénomène et propositions d'orientations » [en ligne], Comité technique Foncier et Développement (AFD-MAEE), juin 2010, consulté en octobre 2011.

URL : http://www.foncier-developpement.fr/wp-content/uploads/appropriation_finale1.pdf LATOUR B., Nous n'avons jamais été modernes. Essai d'anthropologie symétrique, Paris, La Découverte, 1997 (1 ${ }^{\text {re }}$ édition 1991).SANTOS B. DE SOUSA, « Épistémologies du Sud », Études rurales, n 187, 2011 : 21-50. 
TEYSSIER A., L. RAMAROJOHN et R. ANDRIANIRINA RATSIALONANA, « Des terres pour l'agro-industrie internationale ? Un dilemme pour la politique foncière malgache », EchoGéo [en ligne], $n^{\circ} 11,2010$, consulté en octobre 2011.

URL : http://echogeo.revues.org/11649

\section{NOTES}

1. Ce contrat a été dévoilé par Le Monde du 21 mars 2009, au moment de la chute du gouvernement de M. Ravalomana.

2. Acronyme significant "Société de développement hydro-agricole intégrée ".

3. L. CotULA, Land Deals in Africa: What is in the Contracts?, International Institute for Environment and Development (IIED), London, 2011.

4. Acronyme pour les trois forages pétroliers nommés Ishpingo, Tambococha et Tiputini.

5. Le gouvernement équatorien a fixé le montant à $50 \%$ des bénéfices évalués s'il exploitait le pétrole.

6. G. Fontaine, Gaz et pétrole en Amazonie. Conflits en territoires autochtones, Paris, L'Harmattan, $2010: 182$

7. B. de Sousa Santos, «Épistémologies du Sud », Études rurales, n 187, $2011: 22$.

\section{RÉSUMÉS}

Dans cet article, l'auteur étudie un aspect des changements majeurs qui affectent le foncier et, notamment, les acquisitions massives de terre, tels l'effet de délocalisation du rapport à la terre et l'éviction de fait des habitants du territoire qu'ils occupent et travaillent. On voit se multiplier les projets dans lesquels la terre fait l'objet d'une contractualisation dont les habitants sont exclus. L'article analyse d'abord le projet de Varun International à Madagascar, abandonné depuis dans le sillage de l'affaire Daewoo et de la chute du gouvernement de M. Ravalomanana. Le contrat envisagé entre Varun International et le gouvernement malgache était un projet d'agriculture contractuelle (contract farming), qui concernait 465000 hectares et la création de treize périmètres irrigués permettant de produire 2,8 millions de tonnes de riz paddy et 400000 tonnes de maïs dès la $4^{\mathrm{e}}$ année. Or, ce projet est révélateur pour deux raisons. La première est qu'il organisait l'irresponsabilité sociale de l'entreprise par la multiplication de sociétés-écrans chargées de la libérer des relations avec la population. La seconde est qu'il comportait des clauses économiques iniques, qui conduisaient Varun à contrôler $91 \%$ de la production, et qui aurait conduit les agriculteurs malgaches les plus pauvres à devoir acheter du riz pour leur propre nourriture. Ce projet montre comment on aurait pu aboutir à une délocalisation qui est, en réalité, une dys-localisation, c'est-à-dire un dysfonctionnement du rapport normal au lieu. Ce projet organisait les conditions d'une appropriation de la terre et d'un transfert de production dans un pays qui, par ailleurs, dépend du programme d'aide alimentaire mondial. Les conditions du contrat conduisaient à multiplier les schémas triangulaires, par la création d'intermédiaires censés libérer l'entreprise principale de ses devoirs vis-à-vis des hommes et du sol. Comparant ce projet avec le projet « vertueux » de protection du Parc Yasuni en Équateur, l'auteur montre que le même principe de délocalisation est à l'œuvre. Il est question 
de geler l'exploitation du pétrole dans cet espace à la biodiversité unique au monde, à condition que l'État équatorien soit dédommagé des pertes substantielles que le renoncement à l'exploitation pétrolière lui procurerait. Au terme d'un long débat public, les autorités politiques ont en effet indiqué que le pays accepterait d'y renoncer, et de contribuer à la coresponsabilité environnementale globale si la communauté internationale offrait une compensation financière à hauteur de $50 \%$ des pertes estimées. Des pays ou des institutions internationales ont été invités à assurer ce dédommagement. Jusqu'à présent, le fonds international de garantie a enregistré la participation financière du Chili, de l'Espagne, de la Région de Wallonie (Belgique) et de la fondation latino-américaine Avina. D'autres pays ont manifesté leur intérêt comme le Japon, la Norvège, le Portugal, le Canada, l'Allemagne (une promesse de 50 millions d'euros), l'Italie, le Pérou, la Turquie, les Émirats Arabes Unis sans avoir cependant signé d'engagement ferme. Le Programme des Nations Unies pour le développement (PNUD) a offert sa caution. Ainsi, il y a délocalisation, puisque le sort d'un lieu pourrait dépendre désormais des intentions de personnes n'y habitant pas, et même habitant des lieux qui n'ont rien à voir avec celui-ci. Généralisant le propos, l'auteur évoque la fudiciarisation des relations qui sous-tendent ces schémas. Ce qui est en cause, c'est le montage, par des schémas triangulaires complexes et adossés les uns aux autres, de modes financiers et sociaux d'appropriation qui ôtent aux habitants les droits sur la terre qu'ils habitent, qu'on le fasse à leur détriment (dans le cas du projet de Varun), ou pour leur protection (dans le cas de Yasuni). Un lien est proposé avec la théorie néolibérale des Charter cities qui propose une mondialisation de cette relation triangulaire entre trois fonctions : source, hôte, garant. Ces deux projets, l'un abandonné, l'autre en passe de l'être, sont néanmoins révélateurs d'un phénomène général de délocalisation qui affecte les terres et leurs habitants. La délocalisation ou dyslocalisation est la nouvelle mesure du monde, dans la rupture complète avec le rapport ontologique et géographique qui unissait les hommes à leurs milieux. Aujourd'hui, un des nouveaux critères de mesure est la virtualisation du rapport aux lieux pour permettre des formes souples de redéploiement.

In this article, the author studies an important issue affecting land and, in particular, large-scale land acquisitions: the effects of delocalization on relationships with land and the de facto evictions of residents from regions they live and work in. Today, an increasing number of projects involve contracts over land from which residents are excluded. The article initially discusses the Varun International project in Madagascar, which was abandoned as a result of the Daewoo affair and the collapse of M. Ravalomanana's government. Varun International and the Malagasy government had negotiated the terms of a contract farming agreement covering some 465,000 hectares of land. As part of this project, 13 irrigated sectors would produce 2.8 million tons of paddy rice and 400,000 tons of corn within four years of the agreement being signed. The contract is revealing for two reasons. Firstly, it provided for the company's social irresponsibility by creating numerous shell companies to help the company avoid dealing directly with the population. Secondly, the contract contained several extremely unfair clauses. In particular, Varun would have controlled $91 \%$ of production, thus forcing the poorest Malagasy farmers to buy rice for their own food. This project shows how it is possible for delocalization to become "dyslocalization" - a breakdown in the way in which people normally relate to places. It created the necessary conditions for land appropriation and transfers of production in a country that depends on global food assistance. Under the terms of the contract, triangular structures were established. The intermediaries created freed the main company of its duties to the people and the region. The author then discusses another example of delocalization: the project to save Ecuador's Yasuni National Park. This project aims to stop petrol extraction and protect the park's unique biodiversity by compensating the Ecuadorian government for the substantial losses incurred. After a long public debate, the Ecuadorian government agreed to stop petrol production and contribute to global environmental co-responsibility if the international 
community paid compensation equal to $50 \%$ of estimated losses. Countries and international institutions were invited to contribute to this sum. To date, the trust fund has collected financial contributions from Chile, Spain, the Walloon Region (Belgium) and Avina, a Latin American foundation. Other countries, like Japan, Norway, Portugal, Canada, Germany (which promised $€ 50$ million), Italy, Peru, Turkey and the United Arab Emirates have shown an interest in the project, but have not yet made a firm commitment. The United Nations Development Programme (UNDP) administers the trust fund. In this respect, the project is an example of delocalization, because the park's fate now depends on the intentions of people who do not live there. These people live in places that have nothing to do with the park.The author then discusses the fiduciarisation of relationships underlying these structures. The article calls into question the use of complex and interrelated triangular structures to create modes of social and financial appropriation taking away residents' rights over the land they live on - to their detriment (in the Varun project), or for their protection (in the Yasuni example). He draws a parallel with the neoliberal charter cities theory, where the three roles in this triangular relationship - source, host and guarantor - are globalised. Despite the fact that one project has been abandoned and the other is being abandoned, both projects are evidence of a general delocalization movement affecting land and its residents. Delocalization or dyslocalization is the new measure of the world, reflecting the breakdown of ontological and geographical ties linking men to their environment. Today, the virtualisation of relationships to places has become a new measurement criteria for flexible forms of restructuring.

\section{INDEX}

Mots-clés : foncier, acquisitions massives de terres, délocalisation, mondialisation, Projet Yasuni-ITT

Keywords : land, large-scale land acquisitions, delocalization, globalisation, Madagascar, Ecuador

Index géographique : Madagascar, Équateur

\section{AUTEUR \\ GÉRARD CHOUQUER}

Directeur de recherches au CNRS, INSHS

Rédacteur en chef d'Études rurales

Secrétaire de l'association France International Expertise Foncière (FIEF)

chouquer@club-internet.fr 